\title{
Dynamic simulation of a peristaltic micropump considering coupled fluid flow and structural motion
}

\author{
Qiao Lin ${ }^{1}$, Bozhi Yang ${ }^{2}$, Jun Xie $^{3}$ and Yu-Chong Tai ${ }^{3}$ \\ ${ }^{1}$ Department of Mechanical Engineering, Columbia University, New York, NY 10027, USA \\ ${ }^{2}$ Department of Mechanical Engineering, Carnegie Mellon University, Pittsburgh, \\ PA 15213, USA \\ ${ }^{3}$ Division of Engineering and Applied Science, California Institute of Technology, Pasadena, \\ CA 91125, USA \\ E-mail: q12134@columbia.edu
}

Received 9 June 2006, in final form 24 November 2006

Published 22 December 2006

Online at stacks.iop.org/JMM/17/220

\begin{abstract}
This paper presents lumped-parameter simulation of dynamic characteristics of peristaltic micropumps. The pump consists of three pumping cells connected in series, each of which is equipped with a compliant diaphragm that is electrostatically actuated in a peristaltic sequence to mobilize the fluid. Diaphragm motion in each pumping cell is first represented by an effective spring subjected to hydrodynamic and electrostatic forces. These cell representations are then used to construct a system-level model for the entire pump, which accounts for both cell- and pump-level interactions of fluid flow and diaphragm vibration. As the model is based on first principles, it can be evaluated directly from the device's geometry, material properties and operating parameters without using any experimentally identified parameters. Applied to an existing pump, the model correctly predicts trends observed in experiments. The model is then used to perform a systematic analysis of the impact of geometry, materials and pump loading on device performance, demonstrating its utility as an efficient tool for peristaltic micropump design.
\end{abstract}

\section{Introduction}

Micropumps are among the most important microfluidic devices for integrated lab-on-a-chip systems [1-3]. In general, pumps can be divided into two categories: mechanical and non-mechanical pumps. Non-mechanical (e.g., electrokinetic and electrohydrodynamic) pumps can be realized using simple structures without moving parts, but their performance mostly depends on fluid and surface properties [1, 2]. Mechanical pumps, typically employing actuated compliant diaphragms, can handle a large variety of fluids but often involve complicated structures (e.g., as required by check valves) and present integration challenges. Peristaltic micropumps [4-9], however, are a class of mechanical pumps that are relatively simple in structure and amenable to miniaturization and integration. In such devices, a series of diaphragms are actuated in sequence in a manner emulating wave-like muscle contractions of a tube-shaped organ, resulting in forward motion of fluids. We have previously demonstrated a surface-micromachined, electrostatically actuated peristaltic pump [10], which will be the subject of this simulation study.

Simulations of diaphragm-based micropumps that use passive valves have been pursued quite extensively in the literature. While finite element (e.g., [11-14]) and boundary element (e.g., [13, 15]) methods have been used to study the motion of diaphragm actuators in such pumps, lumpedparameter methods have been commonly used to simulate the behavior of complete pumps consisting of diaphragm actuators and check valves. For example, bond graphs have been used, which are based on lumped-parameter energy and power relationships [16]. More commonly, lumped-parameter descriptions of fluid flow and quasistatic diaphragm motion have been employed [17]. These descriptions have also been formulated to represent micropumps in terms of equivalent 


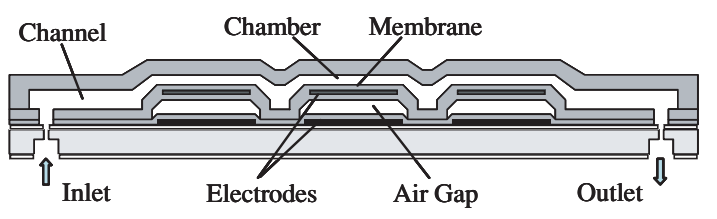

(a)

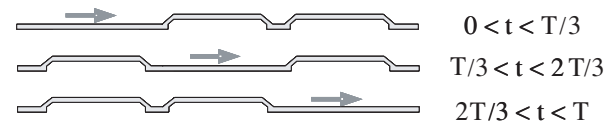

Figure 1. (a) Schematic of the peristaltic pump. (b) Actuation in a period $T$.

electrical networks $[18,19]$. In related work, modeling and simulations have also been reported on pumps consisting of diaphragm actuators and diffuser valves [20-22].

The modeling of peristaltic micropumps, in contrast, has been relatively scarce. Peristalsis theory, developed about 40 years ago $[23,24]$, considers fluid motion in an infinitely long tube whose wall contracts in a prescribed traveling wave form. As such, it does not apply to peristaltic micropumps whose channel wall motion is coupled to fluid flow. Analytical and numerical calculations of static diaphragm deflections and pumping stroke volumes have been considered for piezoelectric peristaltic micropumps [25]. Also restricted to static characteristics, piezoelectric [4] and electrostatic [26] peristaltic pumps have been modeled by assuming the flow rate to be proportional to the actuation frequency and stroke volume, which is determined from the diaphragm motion by ignoring its interaction with fluid flow. More recently, a lumped-parameter analysis has been reported for diaphragmbased micropumps working peristaltically using active valves driven by general actuation schemes [27]. While pumping dynamics is considered, the coupling of viscous fluid flow and diaphragm motion is not adequately accounted for.

This paper presents a simulation study to address the need for understanding the dynamics of peristaltic micropumps involving coupled fluid flow and structural motion. While the basic concept is applicable to general peristaltic micropumps, the study will focus on a surface-micromachined device (figure 1) [10]. Three compliant diaphragms are each located in a thin pumping cell and are actuated electrostatically in a peristaltic sequence to mobilize the fluid. With a preliminary analysis given in [10], we here consider the systematic and in-depth simulation of such a device. Diaphragm motion in each pumping cell is first represented by an effective spring subjected to hydrodynamic and electrostatic forces. These cell representations are then used to construct a systemlevel model for the entire pump, which accounts for both cell- and pump-level interactions of fluid flow and diaphragm vibrations. The first-principle-based model correctly predicts trends observed in experiments and can be evaluated from the device's geometric and material properties without using any parameters that must be experimentally identified. As such, the model can be used as an efficient tool for optimal design of peristaltic micropumps.

The paper is organized as follows. We will first describe the design of the electrostatically actuated peristaltic micropump (section 2). Models for individual pumping cells, including diaphragm vibration and fluid flow, are then

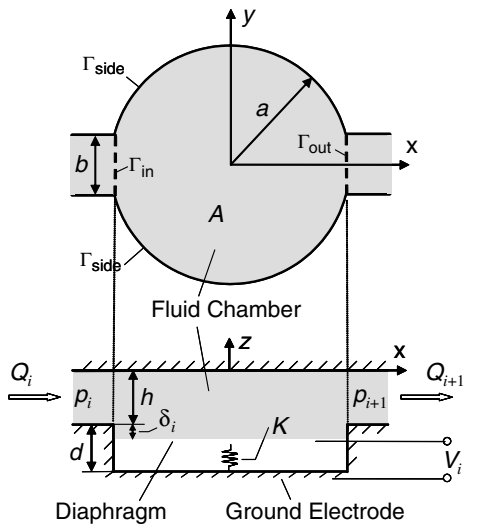

(a)

Figure 2. Representation of a pumping cell.

presented in section 3. The system-level model for the entire micropump is then obtained by linking the models for individual pumping cells (section 4). Modeling results are presented in section 5 and concluding remarks are finally given in section 6 .

\section{Peristaltic pump design}

A schematic of the surface-micromachined peristaltic micropump is shown in figure 1 [10]. The pump has three pumping cells connected in series. Essential components of each pumping cell include a fluid chamber, an electrode gap and a moving diaphragm that separates them. The electrode gap lies between a fixed ground electrode located on the substrate, and a moving electrode embedded in the diaphragm. Air in the electrode gap is vented to the ambient through a venting hole. The fluid chambers in the three pumping cells are connected in series. When an actuation voltage is applied between the two electrodes, electrostatic force will pull the diaphragm downward and cause expansion of the fluid chamber volume. When the pumping cells are actuated using a three-phase peristaltic sequence [6], the resulting peristaltic motion will induce pumping of the fluid inside the chambers. The device is fabricated using parylene-based surface-micromachining technology [28].

\section{Pumping cell representation}

This section considers a representation of the individual pumping cells, which will then be used to construct a model for the entire pump in section 4 . The upstream, middle and downstream pumping cells, including their associated diaphragms, fluid chambers and electrode gaps, are given indices $i=1,2,3$, respectively. They are assumed to have identical shape and dimensions. These include the fluid chamber height $(h)$ and electrode gap thickness $(d)$, both measured in the absence of diaphragm deflection. In addition, the fluid chamber, diaphragm and electrodes are assumed to have the same radius $(a)$, and the channels connected to each chamber have equal width $b$. A representation of the pumping cell $i$ is shown in figure 2. Here, the pressure and flow rate at the entrance of the fluid chamber are, respectively, $p_{i}$ and $Q_{i}$, and those at the exit are $p_{i+1}$ and $Q_{i+1}$. Actuated with a voltage 
$V_{i}$, the diaphragm has deflection $\delta_{i}$, which is represented by the displacement of a spring-attached rigid plate (below). Note that when $V_{i}$ is periodic with frequency $f$, so are the pressure, flow rate and diaphragm deflection. We first consider the diaphragm deflection under electrostatic and hydrodynamic forces and then model the fluid flow in the fluid chamber.

\subsection{Diaphragm vibrations}

The diaphragm in a pumping cell vibrates elastically under the action of electrostatic and hydrodynamic forces. While the deflection varies continuously with location on the diaphragm, for a lumped-parameter representation we approximate the diaphragm as a rigid plate attached to an elastic spring with spring coefficient $K$ (figure 2), whose determination will be described below. The diaphragm deflection is then represented by the displacement of the rigid plate, $\delta_{i}$. This approximation drastically simplifies the description of the diaphragm vibration as well as the associated fluid flow.

In general, in addition to the electrostatic and hydrodynamic forces, the diaphragm vibration is influenced by the effects of diaphragm inertia as well as damping due to air flow in the electrode gap. However, as the electrode gap is connected to the ambient by vent holes, the air flow is largely unimpeded. Thus, air damping effects are small compared to hydrodynamic squeeze-film damping effects (below) and can be neglected. Additionally, vibration theory [29, 30] can be used to estimate that the natural frequency of the diaphragm is about three orders of magnitude higher than the actuation frequency, which is typically below $100 \mathrm{~Hz}$. This implies that the diaphragm inertia is also negligible. Thus, the spring-attached plate is approximately massless and undergoes quasistatic vibration, which is governed by the following algebraic equation:

$$
K \delta_{i}=F_{i}^{\mathrm{elec}}+F_{i}^{\text {hydro }}
$$

where $F_{i}^{\text {hydro }}$ is the hydrodynamic force as will be considered in the next subsection and $F_{i}^{\text {elec }}$ is the electrostatic actuation force given by [31]

$$
F_{i}^{\mathrm{elec}}=\frac{\varepsilon_{0} V_{i}^{2} A}{2\left(d_{\mathrm{eff}}-\delta_{i}\right)^{2}} .
$$

Here, $\varepsilon_{0}$ is the free-space permittivity, $A=\pi a^{2}$ is the approximate diaphragm area and $d_{\mathrm{eff}}=d+t_{p} / \varepsilon_{p}$ is the effective separation of the moving and ground electrodes with $t_{p}$ and $\varepsilon_{p}$ being the thickness and dielectric constant of the insulation layers between the electrodes. For the parylenebased pump, $t_{p}=2 \mu \mathrm{m}$ and $\varepsilon_{p}=3.1$ [10].

The spring coefficient $(K)$, representing the stiffness of the diaphragm, consists of contributions from diaphragm bending, in-plane pretension and stretching of the diaphragm's middle surface [30]. The bending effects are characterized by the plate's bending rigidity, $D=E t_{m}^{3} / 12\left(1-v^{2}\right)$, and pretension by the in-plane force per unit length, $N=\sigma t_{m}$, where $E$ is the Young's modulus, $v$ is Poisson's ratio, $\sigma$ is the in-plane residual stress that results from the temperature history of the fabrication process [32], and $a$ and $t_{m}$ are the diaphragm radius and thickness. For simplicity middle-surface stretching of the diaphragm is neglected [30], so that $K$ is approximately a constant. For the particular case of an electrostatically actuated diaphragm, $K$ can be approximately determined in a way consistent with the determination of the critical voltage, $V_{p}$, at which the electrostatic pull-in instability [31] occurs. That is, while the pull-in voltage is $V_{p}=\left(8 K d_{\mathrm{eff}}^{3} / 27 \varepsilon_{0} A\right)^{1 / 2}$ for the spring-attached rigid plate, it is $V_{p}=\left(4.87 N d_{\mathrm{eff}}^{3} / \lambda \varepsilon_{0} A\right)^{1 / 2}$ for the diaphragm, where $\lambda=1+2(1-\cosh q) /(q \sinh q)<1$ with $q=0.825\left(N a^{2} / D\right)^{1 / 2}$ [33]. We choose the spring coefficient as

$$
K=16.4 N / \lambda
$$

so that these two expressions will consistently give the same pull-in voltage. Note that in the cases $\lambda \rightarrow 1$ and $\lambda \ll 1$, diaphragm stiffness is predominantly due to pretension and bending effects, respectively.

\subsection{Fluid flow}

In a fluid chamber, the diaphragm vibration induces the motion of the thin fluid film confined between the narrow gap formed by the diaphragm and the chamber ceiling. In reality, the fluid film thickness varies continuously with location due to the diaphragm's continuous deflection. However, the approximation of the diaphragm as a spring-attached rigid plate results in a fluid film with uniform thickness, which is only a function of time, $t$. The dynamics of this fluid film is now considered to obtain an expression for the hydrodynamic force $F_{i}^{\text {hydro }}$ (equation (1)) as well as a relationship relating the pressures and flow rates at the entrance and exit of the fluid chamber.

Since the fluid film thickness is small compared with the diaphragm radius (i.e., $h / a \ll 1$ ), its motion can be represented by use of lubrication theory [34]. To begin with, let $f$ be the diaphragm's vibration frequency. Then, the flow velocity in the $z$-direction has a characteristic value of $w_{0}=\omega\left(d_{\text {eff }} / 3\right)$, where $\omega=2 \pi f$ is the circular frequency and $d_{\text {eff }} / 3$, the diaphragm displacement at which electrostatic pull-in instability occurs [33], is taken to be the diaphragm's characteristic displacement. An in-plane characteristic flow velocity can be defined as $u_{0}=w_{0}\left(\pi a^{2}\right) /(b h)$. We next define an out-of-plane Reynolds number $R e_{z}=\rho w_{0} h / \mu$ and an in-plane Reynolds number $R e=\left(\rho u_{0} h / \mu\right)(h / a)=$ $\operatorname{Re}_{z}(\pi a / b)$, where $\rho$ and $\mu$ are the fluid density and dynamic viscosity, respectively. When these Reynolds numbers are of order $h / a$ or smaller, inertial effects in the flow can be ignored when compared with viscous and pressure effects [34]. Also, unsteady flow and gravity effects can be neglected if the squeeze number $\sigma_{s}=\rho \omega h^{2} / \mu=R e_{z}\left(3 h / d_{\mathrm{eff}}\right)$ and the dimensionless parameter $\rho g h^{2} /\left(\mu u_{0}\right)$ are of order $h / a$ or smaller, respectively. When these conditions hold, the classical Reynolds equation of lubrication theory [34] adequately describes the hydrodynamics of the fluid film. In the coordinate frame shown in figure 2 , this equation takes the form

$$
\left\{\begin{array}{l}
\nabla \cdot\left(\left(h+\delta_{i}\right)^{3} \nabla p\right)=12 \mu \dot{\delta}_{i} \\
\left.p\right|_{\Gamma_{\text {in }}}=p_{i},\left.\quad p\right|_{\Gamma_{\text {out }}}=p_{i+1},\left.\quad \frac{\partial p}{\partial n}\right|_{\Gamma_{\text {side }}}=0,
\end{array}\right.
$$

where the pressure, $p$, inside the chamber is assumed not to vary along the fluid film thickness and $\nabla=\partial / \partial x \vec{i}+\partial / \partial y \vec{j}$ is the planar gradient operator. Here, the equation is defined 
over the planar domain corresponding to the fluid chamber, which approximately has the same area, $A$, as the diaphragm. The domain, also denoted as $A$, has its boundary consist of the entrance $\left(\Gamma_{\text {in }}\right)$, exit $\left(\Gamma_{\text {out }}\right)$ and sidewalls $\left(\Gamma_{\text {side }}\right)$ (figure 2$)$. Note that the last boundary condition, in which $\partial / \partial n$ is the derivative along the normal to the boundary, represents the requirement that no fluid penetrates the sidewalls of the chamber.

The fluid film thickness, given by $h+\delta_{i}$ in the presence of diaphragm deflection, does not vary with in-plane coordinates $x$ and $y$. As shown in the appendix, this greatly simplifies the solution of equation (4). In particular, if the fluid chamber is symmetric about the $y$-axes, we obtain the hydrodynamic force

$$
F_{i}^{\text {hydro }}=-\frac{\alpha \mu l^{2} A}{\left(h+\delta_{i}\right)^{3}} \dot{\delta}_{i}+\frac{1}{2} \beta\left(p_{i}+p_{i+1}\right) A
$$

and the flow rates at the chamber inlet and outlet

$$
Q_{i+1}= \pm \frac{1}{2} A \dot{\delta}_{i}+\frac{\gamma\left(h+\delta_{i}\right)^{3}}{12 \mu}\left(p_{i}-p_{i+1}\right)
$$

Here, the coefficients $\alpha, \beta$ and $\gamma$ are given by

$$
\begin{aligned}
& \alpha=\frac{12}{\bar{A}} \int_{\bar{A}} \phi \mathrm{d} \bar{A}, \quad \beta=\frac{2}{\bar{A}} \int_{\bar{A}} \psi \mathrm{d} \bar{A} \text { and } \\
& \gamma=\left.\int_{\bar{\Gamma}_{\text {in }}} \frac{\partial \psi}{\partial \bar{n}}\right|_{\Gamma_{\text {in }}} \mathrm{d} \bar{\Gamma}
\end{aligned}
$$

where the functions $\phi$ and $\psi$ are solutions to the following normalized equations:

$$
\begin{aligned}
& \left\{\begin{array}{l}
\bar{\nabla}^{2} \phi=-1 \\
\left.\phi\right|_{\bar{\Gamma}_{\text {in }}}=0,\left.\phi\right|_{\bar{\Gamma}_{\text {out }}}=0,\left.\frac{\partial \phi}{\partial n}\right|_{\bar{\Gamma}_{\text {side }}}=0
\end{array}\right. \\
& \left\{\begin{array}{l}
\nabla^{2} \psi=0 \\
\left.\psi\right|_{\bar{\Gamma}_{\text {in }}}=1,\left.\psi\right|_{\bar{\Gamma}_{\text {out }}}=0,\left.\frac{\partial \psi}{\partial \bar{n}}\right|_{\bar{\Gamma}_{\text {side }}}=0 .
\end{array}\right.
\end{aligned}
$$

Equations (7) and (8) are given in terms of dimensionless in-plane coordinates $\bar{x}=x / l$ and $\bar{y}=y / l$ with $\bar{\nabla}=$ $\partial / \partial \bar{x} \vec{i}+\partial / \partial \bar{y} \vec{j}$ and $\bar{A}=A / l^{2}$, where $l$ is a characteristic length scale of the fluid chamber domain. Here, $\bar{A}$ also denotes the fluid chamber domain scaled by $l$, with $\bar{\Gamma}_{\text {in }}, \bar{\Gamma}_{\text {out }}$ and $\bar{\Gamma}_{\text {side }}$ denoting the corresponding boundaries scaled by $l$. It is important to note that these coefficients need to be calculated only once for pumping cells of the same shape but different size.

In equation (5), the first term accounts for the squeezefilm damping effects from interactions of viscous fluid flow and diaphragm motion [34] and the second term represents the hydrostatic effects of inlet and outlet pressures. Similarly, in equation (6), the first and second terms account for flow rate contributions from the diaphragm motion and inlet-outlet pressure difference, respectively. It can be observed that in the absence of diaphragm motion, this equation reduces to the classical relationship for Poiseuille flow [35].

Equation (6) provides an integral description of fluid flow in the chamber. Meanwhile, a differential description of diaphragm vibration can be obtained by substituting equation (5) into equation (1):

$$
K \delta_{i}+\frac{\alpha \mu l^{2} A}{\left(h+\delta_{i}\right)^{3}} \dot{\delta}_{i}=\frac{\varepsilon_{0} V_{i}^{2} A}{2\left(d_{\mathrm{eff}}-\delta_{i}\right)^{2}}+\frac{1}{2} \beta\left(p_{i}+p_{i+1}\right) A .
$$

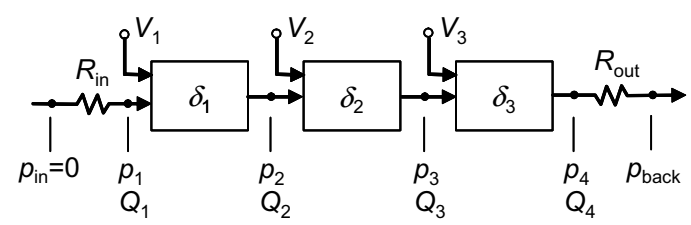

Figure 3. System-level model for the entire peristaltic pump.

\section{System-level pump model}

We can now represent the entire peristaltic pump as a system. This system consists of components including the individual pumping cells and loads connected in series, whose behavior is given by component-level representations described in the previous section. Specifically, each individual pumping cell $i(i=1,2,3)$ is represented by equations (6) and (9), which relate six basic variables and parameters characterizing fluid flow and diaphragm motion in the cell: $p_{i}, p_{i+1}, Q_{i}, Q_{i+1}, \delta_{i}$, and $V_{i}$. The loads upstream and downstream of the pump are typically in the form of microchannels. Neglecting unsteady flow effects and provided the channel length is long compared with the channel's cross-sectional dimensions, such channels can be represented respectively as pure flow resistances, $R_{\text {in }}$ and $R_{\text {out }}$, at the pump inlet and outlet. These flow resistances, based on elementary considerations of Poiseuille flow [35], relate the pressures and flow rates by the following algebraic equations:

$$
-p_{1}=R_{\text {in }} Q_{1} \quad \text { and } \quad p_{4}-p_{\text {back }}=R_{\text {out }} Q_{4}
$$

where $p_{\text {back }}$ is the back pressure at the outlet. The pressure at the pump inlet is set to zero without loss of generality.

The entire pump is thus modeled by equations (6), (9) and (10). As shown in figure 3, the model can be represented using a diagram and corresponds to a system of 11 algebraic and nonlinear ordinary differential equations for the pressures, flow rates and diaphragm deflections which can be readily solved. Note that the model is based on first principles and can be evaluated directly from the device's geometric and material properties without using any experimentally identified parameters.

\section{Results and discussion}

This section presents simulation results obtained from the model obtained in the previous sections. We first apply the model to a device that has been previously tested [10] and use the modeling results to predict the trend in the experimental data. We then use the model to perform a systematic parametric analysis of the impact of geometry, materials and pump loading on device performance.

In all simulations, which are performed using MATLAB [36], the pump is actuated with a three-phase square-wave voltage (figure 1):

$$
\begin{aligned}
& V_{i}=V\left(t-\frac{i-1}{3} T\right) \text { with } \\
& V(t)= \begin{cases}V_{\max } & \text { if } 0<t<T / 3 \text { or } 2 T / 3<t<T \\
0 & \text { if } T / 3<t<2 T / 3\end{cases}
\end{aligned}
$$




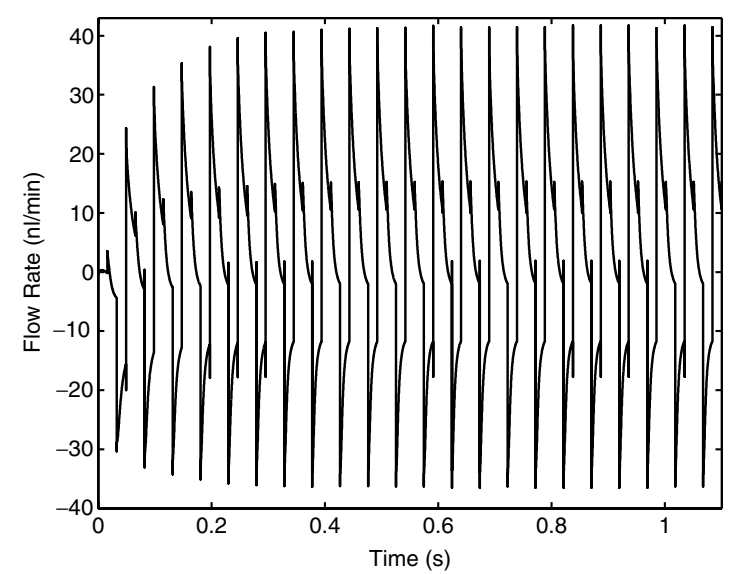

Figure 4. Time course of the flow rate at the pump outlet $\left(Q_{4}\right)$.

where $i=1,2,3$ is the pumping cell index and $T=1 / f$ is the period of the voltage. We assume for simplicity that the pumping loads consist of identical microchannels upstream and downstream of the pump, that is, $R_{\text {in }}=R_{\text {out }}=R_{L}$. The following properties will be used: $E=3.2 \mathrm{GPa}, v=$ $0.4, \sigma=20 \mathrm{MPa}$ and $\varepsilon_{p}=3.1$ for the parylene diaphragm $[10,32]$. Consistent with experiments of [10], the liquid being pumped is taken to be ethanol $\left(\rho=789 \mathrm{~kg} \mathrm{~m}^{-3}\right.$ and $\mu=1.2 \times$ $\left.10^{-3} \mathrm{~Pa} \mathrm{~s}\right)$.

We first consider the modeling of the previously tested pump [10]. The device's dimensions are $a=100 \mu \mathrm{m}, b=$ $60 \mu \mathrm{m}, t_{m}=2 \mu \mathrm{m}$ and $t_{p}=2 \mu \mathrm{m}$. To obtain the spring coefficient using equation (3), we calculate $\lambda=0.81$. This gives $K=815 \mathrm{~N} \mathrm{~m}^{-1}$, which is mostly due to the in-plane tensile residual stress. Primarily due to diaphragm deflections that occur in the fabrication process, the initial fluid chamber height and electrode separation are more difficult to determine and are estimated to be $h=0.9 \mu \mathrm{m}$ and $d=2 \mu \mathrm{m}$. The estimated pumping loads are $R=R_{0}=0.407 \mathrm{kPa}(\mathrm{nl} \mathrm{min})^{-1}$. The actuation voltage amplitude is $V_{\max }=140 \mathrm{~V}$. Choosing $l=\sqrt{A}=\sqrt{\pi} a$, we obtain $\alpha=2.611, \beta=1.000$ and $\gamma=0.6175$ from equations (7) and (8).

For this pump, it can be verified that the assumptions and approximations described in sections 3 and 4 are valid. The model, given by equations (6), (9) and (10), is thus used to study the dynamic behavior of the pump, with the results presented in figures 4 and 5. The back pressure has been set to zero, consistent with the experiment [10]. The time course of the flow rate at the pump outlet $\left(Q_{4}\right)$ is shown in figure 4, with the initial conditions set such that the diaphragms are initially at rest in their undeformed equilibrium positions. The actuation frequency is $f=20.3 \mathrm{~Hz}$, which is actually the optimal frequency for the pump (below). From the figure it can be seen that the flow rate reaches steady state within a fraction of a second. This time scale is determined by the diaphragm compliance and hydrodynamic damping due to the squeezefilm effects and pump loading. Additionally, the flow rate exhibits large fluctuations $\left(41.7 \mathrm{nl} \mathrm{min}{ }^{-1}\right)$ about the average value $\left(1.7 \mathrm{nl} \mathrm{min}^{-1}\right)$. This is characteristic of peristalsis: as the diaphragms are sequentially actuated, the diaphragms do not completely close the fluid chambers. Therefore, the motion of a downstream diaphragm would cause significant back flow in the upstream chambers.

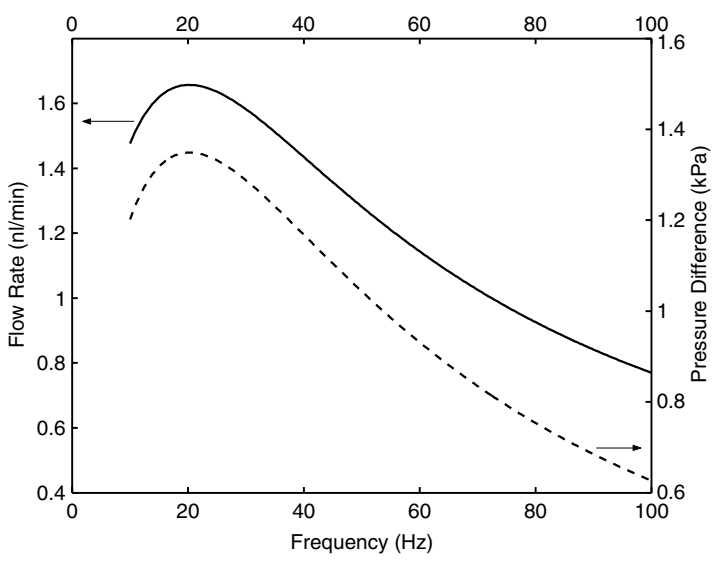

Figure 5. Frequency dependence of the time-averaged flow rate $Q_{\mathrm{av}}$ and the time-averaged value of the pressure difference $p_{4}-p_{1}$.

When the actuation frequency $(f)$ is varied, the timeaveraged flow rate can be computed and compared with experimental observations. The simulation shows that the flow rates $Q_{i}$, for all $i=1, \ldots, 4$, have the same time-averaged value, denoted as $Q_{\mathrm{av}}$, as expected from continuity of fluid flow. This average flow rate is shown in figure 5, along with the time-averaged value of the pressure difference $p_{4}-p_{1}$, which is the pressure head generated by the pump. It can be seen that the average flow rate and pressure both initially increase with $f$ as a result of increasing membrane vibration frequency, respectively reaching the maxima of $1.66 \mathrm{nl} \mathrm{min} \mathrm{m}^{-1}$ and $1.35 \mathrm{kPa}$ at an optimal frequency $f_{\text {opt }}=20.3 \mathrm{~Hz}$. The average flow rate and pressure then steadily decrease as $f$ further increases. This is because at high frequencies the diaphragm vibrations cannot keep up with the excitation and their amplitudes become increasingly diminished. This frequency response is consistent with the trend in the experimental data, in which the average flow rate exhibits a peak value of $1.7 \mathrm{nl} \mathrm{min}^{-1}$ at $20 \mathrm{~Hz}$ [10].

It is also interesting to note that for this pump the voltage amplitude $V_{\max }$ exceeds the static pull-in voltage, which can be calculated (section 3.1) to be $V_{p}=126.7 \mathrm{~V}$. Thus, the diaphragms vibrate beyond the static critical displacement $d_{\text {eff }} / 3=0.88 \mu \mathrm{m}$, at which electrostatic pull-in instability occurs [33]. The vibration is adequately described by our model provided $f$ is greater than a critical value $f_{c}(9.2 \mathrm{~Hz}$ for the pump tested) to prevent membrane touchdown on the fixed ground electrode. We call $f_{c}$ the electrostatic instability bandwidth; when $f<f_{\mathrm{c}}$, electrostatic instability occurs and the model ceases to be valid.

Having considered a particular set of experimentally used pump design parameters, we now perform a systematic investigation of how variations of these parameters impact the performance of the pump. This parametric study will focus on the time-averaged flow rate $Q_{\mathrm{av}}$ as a function of actuation frequency $f$. From dimensional analysis [35] of the governing equations (6), (9) and (10), it can be shown that the parametric dependence of this functional relationship can be most generally studied by varying $b, h, d, R$ and $p_{\text {back }}$ while holding $K, \mu, a$ and $V_{\max }$ fixed at the experimentally used values above. For simplicity, we focus on pump designs that have the same shape as the tested pump, by also keeping $b / a$ 


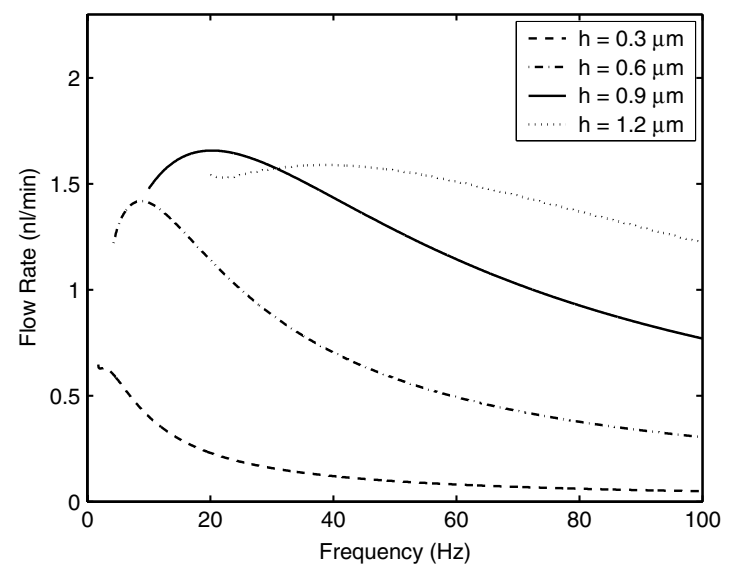

Figure 6. Effects of the variation of the initial fluid chamber height, $h$, on the time-averaged flow rate as a function of actuation frequency.

constant (figure 2). Thus, we will consider below $Q_{\mathrm{av}}$ as a function of $f$ as $h, d, R$ and $p_{\text {back }}$ are varied from the values corresponding to the tested device.

The effect of the initial fluid chamber height $h$ is first considered in figure 6. It can be seen that as $h$ increases, $f_{\text {opt }}$, the optimal frequency at which $Q_{\mathrm{av}}$ achieves its peak value, steadily increases as a result of decreased damping on membrane vibrations. At relatively small $h$, the maximum flow rate initially increases and then decreases with $h$. This is due to the competing effects of increased squeeze-film damping on membrane vibrations and reduced backward flow, both resulting from the decrease in $h$. Additionally, as $V_{\max }>$ $V_{p}$, reduced damping effects due to an increasing $h$ also imply that the diaphragms must vibrate at higher frequencies to avoid touching the bottom electrode. Thus, the electrostatic instability bandwidth $f_{c}$ increases consistently with $h$. Further, it can be observed that $Q_{\mathrm{av}}$ has local minima at 2.1 and $21.5 \mathrm{~Hz}$ for $h=0.3$ and $1.2 \mu \mathrm{m}$, respectively. These minima, which are relatively insignificant, possibly arise from the strong nonlinearities due to squeeze-film damping as well as proximity of the deflected diaphragms to the bottom electrodes.

The effect of the initial electrode separation $d$ is next investigated (figure 7). These results show that the maximum flow rate decreases with $d$, as a larger electrode separation (with $V_{\max }=140 \mathrm{~V}$ unchanged) gives a smaller actuation force. As $d$ decreases from 2 to $1.8 \mu \mathrm{m}$, the pull-in voltage $V_{p}<V_{\max }$ further decreases, leading to a larger electrostatic instability bandwidth $f_{c}$. The optimal frequency, expected to be smaller than $f_{c}$, cannot be computed for the case $d=$ $1.8 \mu \mathrm{m}$ as diaphragm touchdown on the bottom electrode is not validly represented by the model. For $d=2.2$ and $2.5 \mu \mathrm{m}, V_{\max }<V_{p}$ and the pump operation is always stable. It is interesting to observe that the optimal frequency $f_{\text {opt }}$, calculated for $d=2,2.2$ and $2.5 \mu \mathrm{m}$, is not significantly influenced by $d$. This is because $d$ does not directly influence the hydrodynamic force on the diaphragm (equation (5)), but rather only slightly affects the attenuation of diaphragm vibrations by modifying the diaphragm's effective stiffness through the nonlinear dependence of the electrostatic force on diaphragm deflection (equation (2)).

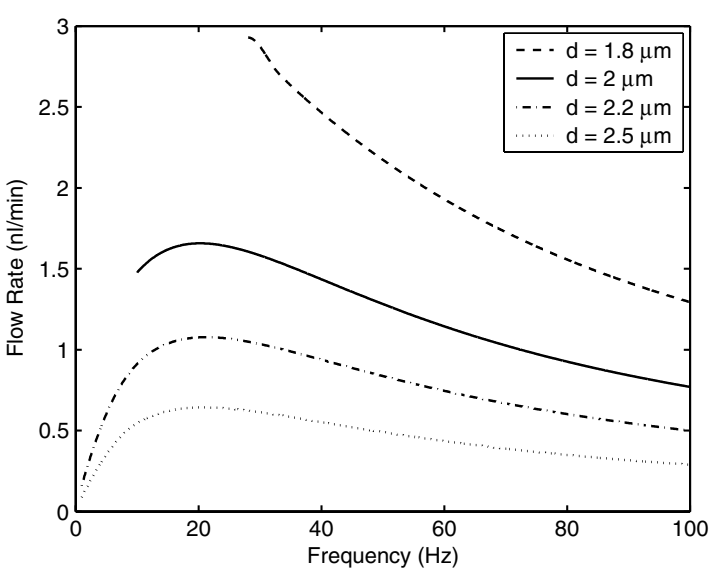

Figure 7. Effects of the variation of the initial electrode separation $d$ on the time-averaged flow rate as a function of actuation frequency.

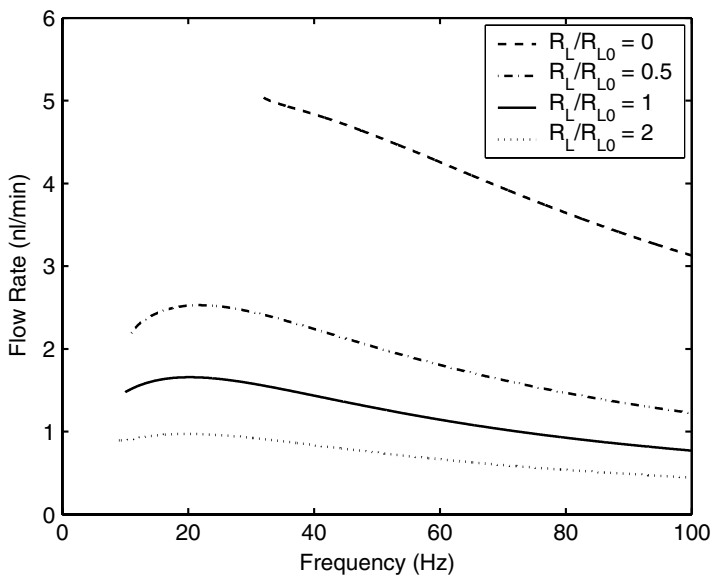

Figure 8. Effects of the pumping load resistance on the time-averaged flow rate as a function of actuation frequency.

We next consider the effect of pumping load $R_{L}$ on the frequency-dependent average flow rate. Figure 8 depicts $Q_{\mathrm{av}}$ as a function of $f$, for different $R$ that is given relative to the load $\left(R_{L 0}\right)$ used in the experiment. It can be seen that the flow rate increases consistently with decreasing load resistance as expected. Decreases in $R_{L}$ also cause a larger electrostatic instability bandwidth $f_{c}$. This is because the decreased pumping load implies that, similar to the case of increasing initial fluid chamber height, the hydrodynamic damping on diaphragm vibrations is reduced and higher actuation frequencies are needed to avoid diaphragm touchdown on the bottom electrode. For $R_{L}=0, f_{c}$ is maximal and $f_{\text {opt }}$ cannot be computed from the model. For the other load resistance values, the calculated $f_{\text {opt }}$ decreases moderately with $R_{L}$ as a result of increased damping on diaphragm vibrations.

Finally, we examine the effects of back pressure on the performance of the pump (figure 9). It can be seen that either in the presence $\left(R_{L}=R_{L 0}\right)$ or absence $\left(R_{L}=\right.$ 0 ) of a pumping load the time-averaged flow rate decreases with the back pressure. Also, at sufficiently large back pressures, the flow rate direction is reversed, as can be seen for $p_{\text {back }}=2 \mathrm{kPa}$ with $R_{L}=R_{L 0}$, indicating that the forward flow generated by peristalsis is insufficient to overcome the back flow generated by the back pressure. We also observe that 


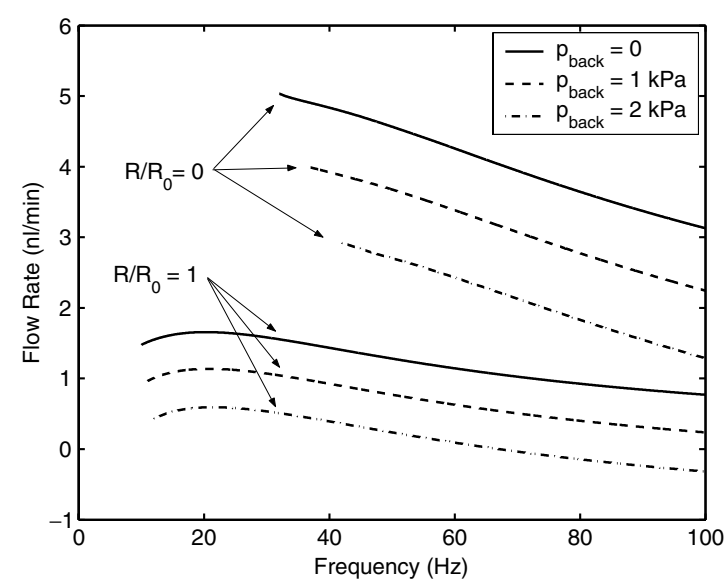

Figure 9. Effects of the variation of back pressure on the time-averaged flow rate as a function of actuation frequency.

the back pressure generates a dc component of the diaphragm deflection towards the bottom electrode and hence causes the electrostatic actuation instability bandwidth $f_{c}$ to consistently increase.

\section{Conclusions}

We have presented a simulation study of a surfacemicromachined peristaltic micropump, which consists of three pumping cells each having an electrostatically actuated diaphragm. Diaphragm motion in each pumping cell is first represented by an effective spring-attached plate subjected to hydrodynamic and electrostatic forces. These cell representations are then used to construct a system-level model for the entire pump, which accounts for both cell- and pumplevel interactions of fluid flow and diaphragm vibrations. As the model is based on first principles, it can be evaluated directly from the device's geometric and material properties without using any parameters that must be experimentally identified. Therefore, the model allows for fast, quantitative determination of the pump's dynamic characteristics and can be used as an efficient tool for optimal design of peristaltic micropumps.

The model has been applied to simulate a surfacemicromachined peristaltic pump that has been experimentally tested. The results from this simulation have revealed bidirectional flow rate fluctuations with amplitudes that are about an order of magnitude larger than the time-averaged flow rate. These fluctuations can be attributed to the pulsatile nature of diaphragm motion as well as backward leakage flow caused by the inability of the diaphragm motion to completely close the fluid chambers. It has also been observed that the timeaveraged flow rate and pumping pressure depend strongly on the actuation frequency $f$. They initially increase with $f$ as a result of increasing membrane vibration frequency, each reaching a maximum $\left(1.66 \mathrm{nl} \mathrm{m^{-1 }}\right.$ and $1.35 \mathrm{kPa}$, respectively) at an optimal frequency $f_{\text {opt }}=20.3 \mathrm{~Hz}$ and then steadily decrease with $f$ due to diminished membrane vibration amplitude. This frequency dependence correctly predicts the trend in the experimental observations [10], suggesting that the model correctly describes the physics of the device.
The model, expressed in geometric and operating parameters, has allowed a systematic analysis of the impact of geometry, materials and pump loading on device performance. This parametric study has yielded the following general observations. First, as the fluid chamber height $h$ increases, the maximum value of the time-averaged flow rate $Q_{\text {av }}$ initially increases and then decreases, as a result of the competing effects of reduced damping on membrane vibrations and increased backward flow. Deceased damping also leads to the steady increase of the optimal frequency $f_{\text {opt }}$ with $h$. Second, as the electrode separation $d$ is decreased, $Q_{\text {av }}$ increases, although $f_{\text {opt }}$ does not vary significantly. This is because a smaller electrode separation for a given actuation voltage amplitude affords a larger actuation force, while $d$ does not directly affect the hydrodynamic damping on the diaphragm. Third, $Q_{\mathrm{av}}$ increases consistently with decreasing pump load resistance as expected, with $f_{\text {opt }}$ increasing moderately due to reduced damping on diaphragm vibrations. Finally, $Q_{\mathrm{av}}$ decreases with the back pressure. At sufficiently large back pressures, the flow rate direction is reversed, indicating that the forward flow generated by peristalsis is insufficient to overcome the back flow generated by the back pressure.

Building on the work presented in this paper, future work can be pursued in several directions. First, the accuracy or efficiency of the model can be further improved. For improved accuracy, it will be necessary to consider the continuous diaphragm deflection and the resulting spatial dependence of the fluid film thickness in a pumping cell. This could be accomplished by numerically solving a coupled system of partial differential equations governing diaphragm vibrations and fluid flow. This would likely only require two-dimensional discretization of the fluid chamber domain and therefore be reasonably efficient. Inclusion of post-pull-in electrostatics of diaphragm motion is another possibility for improving the model accuracy [37]. Improved model efficiency, on the other hand, could be accomplished with the development of a full analytical model. Such a model, which could be obtained by linearization approximations, would also give more intuitive insights into the influence of various parameters on the pump performance, as the role of such parameters can be easily inspected or evaluated from closed-form solutions. Finally, future work will pursue more extensive experimental validation. Due to limited experimental data available for surface-micromachined electrostatic peristaltic pumps, we have focused on using the model to examine the trend observed in the experiments. To allow more detailed comparisons, peristaltic micropumps can be fabricated according to designs generated from the model and tested to yield experimental data needed. These comparisons could also lead to appropriate correction coefficients to reflect real-world issues not accounted for by the model, thereby making the model more generally applicable.

\section{Acknowledgments}

This work was supported in part by the National Science Foundation (grants CTS-0304568 and CCR-0325344). The authors also wish to thank Dr Y Wang for his assistance in numerical computations. 


\section{Appendix. Details of hydrodynamic force and flow rate computation}

We provide the details of computing the hydrodynamic force (equation (5)) and flow rates (equation (6)) in this appendix. The solution of the Reynolds equation (equation (4)), expressed in terms of dimensionless in-plane coordinates $\bar{x}$ and $\bar{y}$, can be decomposed into the following three subproblems by the principle of superposition:

$$
\begin{aligned}
& \left\{\begin{array} { l } 
{ \overline { \nabla } ^ { 2 } \phi = - 1 } \\
{ \phi | _ { \overline { \Gamma } _ { \text { in } } } = 0 , \quad \phi | _ { \overline { \Gamma } _ { \text { out } } } = 0 } \\
{ ( \partial \phi / \partial \overline { n } ) | _ { \overline { \Gamma } _ { \text { side } } } = 0 , }
\end{array} \quad \left\{\begin{array}{l}
\nabla^{2} \psi=0 \\
\left.\psi\right|_{\bar{\Gamma}_{\text {in }}}=1,\left.\quad \psi\right|_{\bar{\Gamma}_{\text {out }}}=0 \quad \text { and } \\
\left.(\partial \psi / \partial \bar{n})\right|_{\bar{\Gamma}_{\text {side }}}=0
\end{array}\right.\right. \\
& \left\{\begin{array}{l}
\nabla^{2} \psi^{\prime}=0 \\
\psi^{\prime}\left|\bar{\Gamma}_{\text {in }}=0, \quad \psi^{\prime}\right|_{\bar{\Gamma}_{\text {out }}}=1 \\
\left.(\partial \psi / \partial \bar{n})\right|_{\Gamma_{\text {side }}}=0
\end{array}\right.
\end{aligned}
$$

with the pressure given by

$$
p=-\frac{12 \mu l^{2} \dot{\delta}_{i}}{\left(h+\delta_{i}\right)^{3}} \phi+p_{i} \psi+p_{i+1} \psi^{\prime} .
$$

Note that the first two subproblems also appear in equation (8). Here, $\varnothing$ can be interpreted as the pressure in the fluid film, normalized by the factor $-12 \mu l^{2} \dot{\delta}_{i} /\left(h+\delta_{i}\right)^{3}$, generated by the diaphragm motion when the inlet and outlet pressures are set to zero. On the other hand, $\psi$ and $\psi^{\prime}$ are the fluid pressure when the diaphragm is fixed; $\psi$ corresponds to a unit inlet pressure and zero outlet pressure and $\psi^{\prime}$ corresponds to a unit outlet pressure and zero inlet pressure.

The hydrodynamic force, $F_{i}^{\text {hydro }}=\int_{A} p \mathrm{~d} A$, can thus be computed as follows.

$$
\begin{gathered}
F_{i}^{\text {hydro }}=-\frac{\mu l^{2} A}{\left(h+\delta_{i}\right)^{3}} \dot{\delta}_{i}\left(\frac{12}{\bar{A}} \int_{\bar{A}} \phi \mathrm{d} \bar{A}\right)+\frac{1}{2} p_{i} A\left(\frac{2}{\bar{A}} \int_{\bar{A}} \psi \mathrm{d} \bar{A}\right) \\
+\frac{1}{2} p_{i+1} A\left(\frac{2}{\bar{A}} \int_{\bar{A}} \psi^{\prime} \mathrm{d} \bar{A}\right) .
\end{gathered}
$$

The flow rate at the inlet is given by $Q_{i}=-\int_{\Gamma_{\text {in }}} q_{n} \mathrm{~d} \Gamma$ where $q_{n}=-\left[\left(h+\delta_{i}\right)^{3} /(12 \mu)\right] \partial p / \partial n$ is the flow rate across a unit-width section of the boundary $\Gamma_{\text {in }}$ [34]. Substituting equation (A.1) then yields

$$
\begin{aligned}
Q_{i} & =-l^{2} \dot{\delta}_{i} \int_{\bar{\Gamma}_{\text {in }}} \frac{\partial \phi}{\partial \bar{n}} \mathrm{~d} \bar{\Gamma}+\frac{\left(h+\delta_{i}\right)^{3}}{12 \mu}\left(p_{i} \int_{\bar{\Gamma}_{\text {in }}} \frac{\partial \psi}{\partial \bar{n}} \mathrm{~d} \bar{\Gamma}\right. \\
& \left.+p_{i+1} \int_{\bar{\Gamma}_{\text {in }}} \frac{\partial \psi^{\prime}}{\partial \bar{n}} \mathrm{~d} \bar{\Gamma}\right) .
\end{aligned}
$$

Equations (A.2) and (A.3) are valid for a pumping chamber of arbitrary shape. Now consider the special case that the chamber is symmetric about the $y$-axis in the plane. Then, $\psi^{\prime}(\bar{x}, \bar{y})=\psi(-\bar{x}, \bar{y})$ and $\int_{\bar{A}} \psi^{\prime} \mathrm{d} \bar{A}=\int_{\bar{A}} \psi \mathrm{d} \bar{A}$. Using the definitions of the coefficients $\alpha$ and $\beta$ (equation (7)), equation (A.2) reduces to the desired form given in equation (5).

Furthermore, using the symmetry as well as the condition that the flow fields corresponding to the functions $\varnothing, \psi$ and $\psi^{\prime}$ must satisfy continuity at the inlet and outlet, it can be shown that $\int_{\bar{\Gamma}_{\text {in }}} \partial \phi / \partial \bar{n} \mathrm{~d} \bar{\Gamma}=-\bar{A} / 2$ and $\int_{\bar{\Gamma}_{\text {in }}} \partial \psi^{\prime} / \partial \bar{n} \mathrm{~d} \bar{\Gamma}=$ $-\int_{\bar{\Gamma}_{\text {in }}} \partial \psi / \partial \bar{n} \mathrm{~d} \bar{\Gamma}$. Thus, using the definition of the coefficient $\gamma$ in equation (7), equation (A.3) reduces to the desired form for the inlet flow rate $Q_{i}$ given in equation (6). Finally, using $Q_{i}$ and the continuity condition $Q_{i}-Q_{i+1}=A \dot{\delta}_{i}$ yields the outlet flow rate expression $\left(Q_{i+1}\right)$ in equation (6).

\section{References}

[1] Laser D J and Santiago J G 2004 A review of micropumps J. Micromech. Microeng. 14 R35-64

[2] Nguyen N T, Huang X Y and Chuan T K 2002 MEMS-micropumps: a review Trans. ASME, J. Fluids Eng. 124 384-92

[3] Shoji S and Esashi M 1994 Microflow devices and systems J. Micromech. Microeng. 4 157-71

[4] Bu X M, Melvin T, Ensell G, Wilkinson J S and Evans A G R 2003 Design and theoretical evaluation of a novel microfluidic device to be used for Pcr J. Micromech. Microeng. 13 125-30

[5] Cabuz C, Cabuz E I, Herb W R, Rolfer T and Zook D 1999 Mesoscopic sampler based on $3 \mathrm{~d}$ array of electrostatically activated diaphragms Proc. 10th Int. Conf. on Solid-State Sensors, Actuators and Microsystems (Transducers'99) (Sendai, Japan) pp 1890-1

[6] Grosjean C, Yang X and Tai Y C 1999 A thermopneumatic peristaltic micropump Proc. 10th Int. Conf. on Solid-Sate, Sensors, Actuators and Microsystems (Transducers'99) (Sendai, Japan) pp 1776-9

[7] Judy J W, Tamagawa T and Polla D L 1991 Surface-machined micromechanical membrane pump Micro Electro Mechanical Systems, 1991 (MEMS'91) Proc.: An Investigation of Micro Structures, Sensors, Actuators, Machines and Robots (IEEE) pp 182-6

[8] Kai E, Pan T and Ziaie B 2004 A robust low-cost PDMS peristaltic micropump with magnetic drive Tech. Dig. Solid-State Sensor, Actuator and Microsystems Workshop (Hilton Head'04) (Hilton Head Island, SC) pp 270-3

[9] Na S, Ridgeway S and Cao L 2003 Theoretical and experimental study of fluid behavior of a peristaltic micropump Proc. 15th Biennial IEEE University/Government/Industry Microelectronics Symp. (Boise, Idaho) pp 312-6

[10] Xie J, Shih J, Lin Q A, Yang B Z and Tai Y C 2004 Surface micromachined electrostatically actuated micro peristaltic pump Lab Chip 4 495-501

[11] Gong Q L, Zhou Z Y, Yang Y H and Wang X H 2000 Design, optimization and simulation on microelectromagnetic pump Sensors Actuators A 83 200-7

[12] Gonzalez U F and Moussa W A 2002 Simulation of MEMS piezoelectric micropump for biomedical applications 2002 ASME Int. Mech. Eng. Conf. \& Exp. (IMECE'02) (New Orleans, LA)

[13] Kaltenbacher M, Landes H, Lerch R and Lindinger F 1997 A finite-element/boundary-element method for the simulation of coupled electrostatic-mechanical systems J. Physique III. 7 1975-82

[14] Morris C J and Forster F K 2000 Optimization of a circular piezoelectric bimorph for a micropump driver J. Micromech. Microeng. 10 459-65

[15] Jiang T Y, Ng T Y and Lam K Y 2000 Dynamic analysis of an electrostatic micropump Proc. Int. Conf. Modeling and Simulation of Microsystems (San Diego, CA) pp 632-5

[16] Van der Pol F C, Breeveld P C and Fluitman J H J 1990 Bond graph modeling of an electro-thermo-pneumatic micropump Tech. Dig., Micro Mechanics Europe, 2nd Workshop on Micromachining \& Microsystems pp 19-24

[17] Zengerle R and Richter M 1994 Simulation of microfluid systems J. Micromech. Microeng. 4 192-204

[18] Bourouina T, Bosseboeuf A and Grandchamp J P 1997 Design and simulation of an electrostatic micropump for drug-delivery applications J. Micromech. Microeng. 7 186-8

[19] Bourouina T and Grandchamp J P 1996 Modeling micropumps with electrical equivalent networks J. Micromech. Microeng. 6 398-404

[20] Banerjee D, Yano K and Bart S F 2001 System model for a novel no moving parts diffuser valve based diaphragm actuated micro pump Tech. Proc. 2001 Int. Conf. on 
Modeling and Simulation of Microsystems (MSM'01) pp 578-81

[21] Olsson A, Stemme G and Stemme E 1999 Numerical of flat-walled diffuser elements for valveless micropumps Tech. Proc. 1999 Int. Conf. on Modeling and Simulation of Microsystems (MSM' 99) (San Juan, Puerto Rico) pp 585-8

[22] Ullmann A, Fono I and Taitel Y 2001 A piezoelectric valve-less pump-dynamic model Trans. ASME, J. Fluids Eng. 123 92-8

[23] Fung Y C and Yih C S 1968 Peristaltic transport J. Appl. Mech. 35 669-75

[24] Shapiro A H, Jaffrin M Y and Weinberg S L 1969 Peristaltic pumping with long wavelengths at low Reynolds number J. Fluid Mech. 37 799-825

[25] Cao L, Mantell S and Polla D 2001 Design and simulation of an implantable medical drug delivery system using microelectromechanical systems technology Sensors Actuators A 94 117-25

[26] Teymoori M M and Abbaspour-Sani E 2005 Design and simulation of a novel electrostatic peristaltic micromachined pump for drug delivery applications Sensors Actuators A 117 222-9

[27] Goldschmidtboing F, Doll A, Heinrichs M, Woias P, Schrag H J and Hopt U T 2005 A generic analytical model for micro-diaphragm pumps with active valves $J$. Micromech. Microeng. 15 673-83
[28] Wang X-Q and Tai Y-C 2000 A normally closed in-channel micro check valve 13th Annual Int. Conf. on Micro Electro Mechanical Systems, 2000 (MEMS 2000) pp 68-73

[29] Leissa A W 1993 Vibration of Plates (Melville, NY: Acoustical Society of America)

[30] Timoshenko S and Woinowsky-Krieger S 1959 Theory of Plates and Shells 2nd edn (New York: McGraw-Hill)

[31] Kovacs G T A 1998 Micromachined Transducers Sourcebook (Boston: WCB/McGraw-Hill)

[32] Harder T A, Yao T-J, He Q, Shih C-Y and Tai Y-C 2002 Residual stress in thin-film parylene-C 15th IEEE Int. Conf. on Micro Electro Mechanical Systems, 2002 (MEMS 2002) pp 435-8

[33] Osterberg P M and Senturia S D 1997 M-test: a test chip for MEMS material property measurement using electrostatically actuated test structures J. Microelectromech. Syst. 6 107-18

[34] Hamrock B J 1994 Fundamentals of Fluid Film Lubrication (New York, NY: McGraw-Hill)

[35] White F M 2003 Fluid Mechanics 5th edn (New York: McGraw-Hill)

[36] The MathWorks Matlab ${ }^{\mathbb{B}}$-The Language of Technical Computing (http://www.mathworks.com/products/matlab)

[37] Rocha L A, Cretu E and Wolffenbuttel R F 2004 Behavioural analysis of the pull-in dynamic transition $J$. Micromech. Microeng. 14 S37-42 